\title{
De la théorie aux pratiques culturales sur les
} atolls...

Jean-Michel Chazine

\section{OpenEdition}

12 Journals

Édition électronique

URL : http://journals.openedition.org/jso/1390

DOI : $10.4000 /$ jso. 1390

ISSN : $1760-7256$

Éditeur

Société des océanistes

\section{Édition imprimée}

Date de publication : 1 décembre 2002

Pagination : 63-69

ISSN : 0300-953x

\section{Référence électronique}

Jean-Michel Chazine, «De la théorie aux pratiques culturales sur les atolls... », Journal de la Société des Océanistes [En ligne], 114-115 | Année 2002, mis en ligne le 26 mai 2008, consulté le 22 mars 2021. URL : http://journals.openedition.org/jso/1390 ; DOI : https://doi.org/10.4000/jso.1390

\section{(c)}




\section{De la théorie aux pratiques culturales sur les atolls...}

par

Jean-Michel CHAZINE *

\section{RÉSUMÉ}

L'observation des fosses de culture sur les atolls des Tuamotu, puis les recherches sur leur mode de fonctionnement avaient conduit l'auteur à rencontrer Jacques Barrau et à en retrouver des analogies archéologiques. Des investigations et expérimentations ethno-archéologiques vont montrer que de la Micronésie aux Tuamotu de l'Est, ces techniques avaient été élaborées de la même manière, même si leur persistance n'a pas été la même. Les réflexions pionnières de Jacques Barrau ont ainsi été corroborées quelques décennies plus tard.

Mots-CLÉs : Atolls, Pacifique, horticulture, ethnoarchéologie, fosses de culture, taro.

\section{Introduction}

Repenser aux premiers instants de ses recherches, à l'occasion d'un hommage rendu à quelqu'un qui aura eu une influence déterminante, m'a immanquablement fait venir à l'esprit les fameux « je me souviens » de George Perec. Et cet appel à une mémoire enfouie, endormie, mais bien vivace, l'évoque irrésistiblement.

Je me souviens, oui, c'était en 1975, à Tahiti. Je me souviens des nouvelles découvertes archéologiques mais aussi culturelles, des rencontres inattendues, de palanquées de jeunes à l'engouement fluide, des différents organismes de jeunesse, unanimement rameutés pour l'occasion, je me souviens de nos enthousiasmes et de nos déceptions, des miennes surtout peut-être... Je me sou-

\section{ABSTRACT}

The observation of cultivation pits on the Tuamotuan atolls followed by researches upon their utilisation process had led the author to meet Jacques Barrau and find out some archaeological analogies. Further investigations and ethnoarchaeological experiments will prove that from Micronesia to Eastern Tuamotu, these technics had been alaborated upon the same trend, even if their persistance had not been the same. Jacques Barrau's pionneering thoughts have thus been confirmed some decades later.

Keywords : Atolls, Pacific, horticulture, ethnoarchaeology, cultivation pits, taro.

viens, c'était la seconde année où José Garanger m'avait proposé de venir continuer l'énorme travail archéologique entrepris par lui à Tahiti, sur le marae Ta'ata à Paea d'abord, et dans la petite et prolifique vallée de la Vaiote, au bout de la presqu'île ensuite. Je me souviens aussi que ces activités s'étaient plutôt bien déroulées et qu'il s'y était ajouté, de manière imprévue, une prospection dans la vallée de la Papeno'o, à la suite d'un premier repérage de Bertrand Gérard, à cause d'un projet de construction de barrage qui allait ennoyer une grande portion de la vallée médiane. Et puis, il y eut cette soudaine mais insistante proposition de Paul Moorgat, infatigable homme lige de la Société des Études Océaniennes, suscitée par le programme «Man and Biosphère » (MAB, 1975) de l'UNESCO, mis en

* CNRS : CREDo/Maison Asie-Pacifique Marseille. 
place par Bernard Salvat, d'aller en mission aux Tuamotu, et en particulier à Takapoto.

\section{Les fosses de culture : prise de contact, prise de conscience}

L'objectif était de tenter de reconstituer, à partir des données de l'archéologie de surface, le processus de peuplement de cet atoll au cours du temps. J'avais eu l'année précédente un petit aperçu expérimental d'un mini-atoll, celui de Taiaro, sur lequel pendant plus d'une semaine d'investigations, nous n'avions quasiment rien découvert ni pu réussir à observer de l'ancienne culture traditionnelle. Aussi, mon expérience réelle des îles basses et de leur occupation passée se réduisait-elle à quelques lectures, et plus précisément celle des travaux sur Rangiroa de J. Garanger et A. Lavondès d'une part (1967) et, plus superficiellement, de Paul Ottino (1972) d'autre part, les uns et les autres, forcément et fortement inspirés par les observations pionnières dans les années 1920-1930 de K.P. Emory, du B.P. Bishop Museum de Hawaii.

De ces lectures préalables et un peu prémonitoires, m'était restée la conscience que ces îles basses, au sol ingrat et aux ressources limitées, permettaient à peine la survie de leurs occupants, eux-mêmes déjà rescapés d'îles hautes et lointaines. De ces survols plutôt que de véritables lectures assidues, j'avais cependant noté «qu'ils», ces pauvres insulaires déshérités, avaient utilisé des fosses de culture pour cultiver essentiellement des tubercules, en particulier des taros. La description physique de ces espaces pourtant dévolus à une horticulture assez élaborée restait néanmoins assez floue et difficilement imaginable.

J'avais probablement aussi, sinon lu, tout au moins parcouru également plus ou moins superficiellement « Subsistence Agriculture in Polynesia and Micronesia » de Jacques Barrau (1961), afin d'avoir des notions au moins sommaires sur une horticulture océanienne encore assez éloignée des observations archéologiques que je commençais à engranger. Pour une connaissance plus générale, j'avais aussi déjà lu la compilation établie par A. P. Vayda (1968) grâce à laquelle les préoccupations des anthropologues-environnementalistes anglo-saxons avaient commencé à m'être plus familières.

Arrivé à Takapoto pour un séjour d'à peine deux semaines, je ne disposais pratiquement pour tout élément d'information ou de localisation sur place que de la vague carte des relevés qu'Emory y avait faits et de l'expérience sympa- thique mais néanmoins vigilante et distanciée de Dany Carlson, alors responsable locale du Service de la Pêche mais, surtout, passionnée de culture polynésienne et paumotu, ayant participé auparavant à de fructueux travaux archéologiques, tant avec K.P. Emory qu'avec Y. Sinoto.

C'est là qu'au quatrième ou cinquième jour, en continuant d'élaborer mes techniques et stratégies de prospection, je me suis surpris à parcourir une assez grande zone de montagnes russes de 4 à $5 \mathrm{~m}$ de dénivellation. Il me fallut en monter et descendre successivement plusieurs pour que je finisse par prendre physiquement et même littéralement conscience de ce qu'étaient réellement ces fosses de culture : une succession sur près de $100 \mathrm{~m}$ de longueur et 15 à $20 \mathrm{~m}$ de largeur, de buttes et de creux alternés. La zone venait d'être " nettoyée », c'est-à-dire totalement brûlée et débarrassée de ses adventices et, comme c'était le matin, le relief en était nettement visible. Les parois de ces fosses laissaient apparaître par endroit le substrat corallien clair : sables, granulats et même grès de plage, alors que les fonds étaient manifestement brun-sombre à noirs. Des nappes boueuses percées de nombreux trous de tupa (Carnifex carnissonis) s'étalaient même en de nombreux endroits. De formes plutôt géométriques allongées ou oblongues, ces fosses couvraient une surface au sol assez importante et, surtout, le volume des déblais qui leur correspondait était particulièrement impressionnant, comparé à la planéité généralisée de la surface de l'atoll.

C'est ce contraste là qui m'a tout d'abord frappé, ensuite seulement le côté paradoxal, voire dérisoire, des outils qui avaient pu ou dû être employés pour creuser ces fosses. Avais-je déjà lu que c'est en utilisant seulement des nacres ou des fragments de carapace de tortues emmanchées qu'elles avaient été creusées? Probablement pas, et je ne le sais plus exactement maintenant. La publication en anglais cette même année de Emory (1975) qui allait devenir, pour nous tous, un ouvrage de référence sur la culture matérielle passée des Tuamotu, n'était pas encore arrivée à Tahiti et je ne pouvais en avoir encore vu les images. Le fait est que j'ai rapidement observé et récolté plusieurs fragments de coquilles de nacre présentant des perforations qui m'ont fait penser qu'elles avaient été utilisées et montées de cette manière ( $c f$. Chazine, 1977 : 205-215).

C'est à partir de ce moment que je me suis rendu compte de l'insuffisance des observations qui avaient été faites par rapport à la quantité de 
travail individuel mais aussi probablement collectif, qui avait été manifestement mise en œuvre là, tout autant que la quantité de production vivrière que ces fosses avaient visiblement pu fournir. Le potentiel informationnel qui m'était soudain apparu ne pouvait plus dès lors être négligé ni sous-évalué. On avait directement sous les yeux les vestiges des jardins potagers des anciens occupants des atolls; on pouvait en calculer toutes les surfaces, tout le potentiel productif, en déduire quelle taille avaient les communautés qui avaient pu s'en être nourrie et en extrapoler même l'exacte quantité de travail voire de travailleurs qui les avaient creusées. Une mine rare si ce n'est unique pour des investigations archéologiques...

À partir de ce moment, décompter, localiser et analyser la morphologie des différents marae de cet atoll, puisque c'étaient a priori les seules structures manifestement apparentes et liées à une organisation socio-religieuse antérieure, m'a immédiatement semblé limité et, surtout, peu représentatif d'autres préoccupations, activités ou productions aussi essentielles qu'importantes. À dire vrai, je m’y suis astreint mais, en fait, je trouvais cette démarche par trop restrictive et surtout un peu vaine. La réalité passée des atolls pouvait-elle être, ne serait-ce même qu'incomplètement, déduite et reconstituée à partir d'un complément d'inventaire de ses structures religieuses? Complément aussi précisément descriptif qu'il soit possible? Il est pourtant vrai et quelque peu paradoxal que cet inventaire des structures lithiques, qu'elles soient encore présentes matériellement ou dans la mémoire des gens, faisait - et paradoxalement - fait encore partie, près de trente ans plus tard, de mes/nos programmes de recherches sur les îles basses. Ce fut le cas dans l'important travail de prospection et de relevés effectué dans le programme initié et conduit par S. Hatanaka à Reao en 1976 d'abord par Y. Sinoto puis en 1980 par E. Nitta et moimême qui avons pu localiser près de 85 marae (Hatanaka and Shibata, 1978 et 1982). Pareillement en 1985, à la suite de la mission réalisée par É. Conte et $\mathrm{J}$. Tchong où 40 à 56 marae inédits furent étudiés ou découverts, sans qu'une grille d'interprétation réellement nouvelle ou fonctionnelle de ces données complémentaires puisse en être déduite (Conte, 1990: 78-86). Il faut pourtant continuer à penser que l'établissement à terme de typologies spatio-temporelles, de variantes et de styles ou de procédures techniques d'une extrémité à l'autre de l'archipel ne serait-ce que des seules îles des Tuamotu sera possible et fournira certainement des données utiles pour préciser au moins les aires d'influence ou de diffusion culturelles ou coutumières.

\section{Une rencontre déterminante : Jacques Barrau}

Ainsi ai-je continué la prospection rétrospective de celle d'Emory, complétée par de nouvelles observations, mais en m'attachant à repérer et même comptabiliser avec une plus grande précision les zones de fosses. Les résultats de cette prise de contact, mais aussi, par la même occasion, de conscience, en ont été publiés (Chazine, 1977). C'est la rédaction même de cet article, par les recherches bibliographiques qui l'ont accompagnée qui m'ont alors mis en relation plus personnelle avec Jacques Barrau. J'avais eu l'occasion et le plaisir de l'écouter lors de séminaires généraux ou ponctuels sur les activités liées à la subsistance en Océanie, avec Godelier, Cresswell ou d'autres, et ma découverte d'une aussi importante architecture agraire sur les atolls nécessitait une rencontre plus personnelle avec lui.

L'accueil et l'écoute qu'il m'a immédiatement apportés ont été à la hauteur de mes espérances. Sa chaleur humaine, modulée par une perception très critique des paramètres sociaux et l'intérêt qu'il a néanmoins manifesté pour ces techniques agraires devenues archéologiques aux Tuamotu, alors qu'il en avait fait des observations ethnographiques en particulier en Micronésie, me restent en mémoire. Il était évident pour lui que sur un espace aussi confiné et réduit qu'une île basse, la présence de ces structures construites en négatif rendait effectivement exactement compte de préoccupations et d'une organisation communautaire importantes. Les mesures que j'avais commencé empiriquement à entreprendre à Takapoto ne pouvaient qu'en compléter d'autres, celles engagées en Micronésie, par exemple.

Il me confia alors ce qui allait devenir comme un exceptionnel cadeau dans lequel j'allais par la suite me plonger pendant longtemps et qui s'est avéré être même déterminant pour la majeure partie de ma vie professionnelle ultérieure. Il disposait en effet de la série complète des anciens numéros de la publication lancée après la guerre des Atoll Research Bulletins dont M. H. Sachet était une des rédactrices principales et qu'il me confia. Je m'immergeai alors grâce à ses conseils dans la reconstitution de cette horticulture oubliée et suivis surtout ses encouragements à continuer sur le thème peu ou même pas du tout encore exploité des fosses de culture et des techniques culturales mises en jeu sur les îles basses océaniennes. 
Ainsi que je l'ai rappelé plus haut, en dehors des travaux extensifs d'Emory, suivis quelque trente ans plus tard par les prospections archéologiques de Garanger et Lavondès, localisées uniquement à Rangiroa car bénéficiant des repérages ethnographiques préalables de Paul Ottino, quasiment rien n'avait été réalisé aux Tuamotu.

La lecture de ces différents Atoll Research Bulletins qui sont une mine de données et d'observations pluridisciplinaires à peu près toutes reliées, en outre, à des observations ethnographiques et contemporaines, de l'après-guerre aux années soixante, m'a ouvert un champ d'investigation et de réflexion dont je n'avais pas idée au départ. Surtout, il me donnait à "voir», en les transposant depuis le Pacifique central, l'état et l'organisation socio-technique possible, si ce n'est probable, des communautés ayant occupé autrefois les atolls des Tuamotu.

Et puis, surtout, cela justifiait la recherche de l'obtention, enfin possible, de données numériques et quantifiées sur les productions de ressources vivrières d'insulaires dont seules les prouesses et connaissances hauturières étaient vantées. Par un va-et-vient entre les reconstitutions théoriques du passé que les méthodes ou observations archéologiques permettaient aux Tuamotu et les données contemporaines de Micronésie notamment, il devenait possible d'y restituer et faire émerger un cadre fonctionnel. Cette dialectique a d'abord en quelque sorte produit une esquisse, devenue par la suite, grâce à quelques témoignages de tradition orale, une véritable synergie archéo-ethnographique consacrée aux îles basses du Pacifique en général.

Pendant la quinzaine d'années qui a suivi, de nombreuses missions dans différents secteurs des Tuamotu ont permis de préciser à la fois la grande fréquence de la présence de ces fosses de culture - encore que leur répartition n'ait pas la régularité de distribution qu'on leur attribuait ou qu'on attendait à priori - tout en recueillant probablement les derniers témoignages vécus ou transmis de l'époque de leur ultime fonctionnement.

Bien que la reconstitution de toutes les procédures et chaînes opératoires tant techniques que socioculturelles qui les organisent, depuis les critères de choix des lieux de creusement jusqu'aux différents processus d'organisation sociale et procédés d'exploitation menant aux récoltes, ait été peu à peu reconstituée puis remontée, c'est la comparaison finale dynamique et vivante avec la Micronésie qui l'entérinera. En effet, une mission d'accompagnement avec la Commission du Pacifique Sud pour le PROE (Programme de
Recherches Océanien sur l'Environnement), en 1989, me permettra de visiter plusieurs atolls de Micronésie (Majuro, Tarawa) et de Polynésie (Funafuti). Les observations que j'ai pu y faire, au-delà de quelques différences culturelles ou coutumières, ont parfaitement corroboré les déductions que j'avais pu dégager de l'ensemble de mes recherches sur les Tuamotu. Là, j'ai effectivement eu l'impression de faire un saut dans le temps et de « retrouver » les Tuamotu tels qu'ils avaient pu être un ou deux siècles auparavant. Une sensation que A. Guilcher avait lui-même éprouvée en 1967 lors d'un séminaire sur les problèmes de santé et d'écologie dans les atolls, organisé par la Commission du Pacifique Sud à Tarawa, en écrivant: "Les Gilberts, c'est le vieux Pacifique, les Tuamotu d'autrefois, mais certes pas celles d'aujourd'hui"' (Guilcher, 1967 : 109). Bien qu'il se soit basé sur des thèmes d'observations d'ordre ethnographique simples, il avait pu observer « la persistance de la culture du taro en fosses », en rappelant que « la culture en fosses a été autrefois largement répandue, notamment aux Tuamotu, mais son aire s'est beaucoup restreinte de nos jours, et sa conservation aux Gilberts peut être considérée comme un archaïsme ». Un archaïsme qui ne peut que faire, au moins, le bonheur d'un ethno-archéologue.

\section{Établissement d'un corpus de données aux Tuamotu}

Ce type de survivance, et surtout les modalités de son maintien, sont pratiquement tributaires des conditions d'isolement et des ressources économiques disponibles, plus que de la solidité des traditions socioculturelles qui les organisent. Ainsi, aux Tuamotu, c'est dans les atolls les plus isolés que l'utilisation de ces fosses a perduré le plus longtemps ; de même, est-ce souvent là aussi que des témoins directement occulaires ou par ouï-dire ont pu être retrouvés. Ce sont donc vers ces îles, pour autant que l'accès en était possible que nous avons orienté nos recherches.

Plusieurs programmes qui ont obtenu l'aval et le soutien financier d'abord de la CORDET, à partir de 1982, puis de l'ATP (Association Thématique de Programme) «Dynamique des systèmes agraires » du Ministère de la recherche en 1984, suivis eux-mêmes en 1988 de celle portant sur "L'histoire de l'Environnement », ont permis, avec les soutiens logistiques locaux tant du Département Archéologie que du Service de l'Agriculture puis de l'orstom à Papeete, de conduire des investigations à la fois ethnoarchéologiques mais aussi agro-technologiques 
extensives et surtout inédites en Polynésie française.

C'est ainsi que les analyses des sols des fonds de fosse ont confirmé un enrichissement, non seulement en matières organiques provenant des amendements en végétaux soigneusement choisis et fragmentés, mais également en apports minéraux, eux aussi apportés par cette sélection de végétaux et leurs sols, parfaitement bien sélectionnés. De même, l'observation par les insulaires que nombre d'oiseaux nichaient fréquemment dans les ramures du Pisonia grandis leur a fait utiliser non seulement les feuillages broyés de cet arbre, mais aussi la couche superficielle du sol sous-jacent, lui-même fortement concentré en phosphates par leurs fientes. Ces mêmes observations ont d'ailleurs été confirmées par la suite à Kiribati (Di Piazza, 2001). Une autre plante, par exemple, l'algue hydrophile (Gleocapsia algicola) permet de fixer l'azote. Les analyses qui ont été faites indiquent un enrichissement de 20 à $30 \%$. Les connaissances empiriques accumulées au cours de leur séjour sur ces îles par les insulaires sont ainsi particulièrement adaptées et complexes.

Les premiers décomptes ou estimations portant sur les surfaces, les formes, les répartitions de celles-ci, entre elles ainsi que comparées à leur distribution sur le pourtour des atolls, ont alors été systématiquement effectués, partout où l'on a séjourné. L'expérience venant et les techniques de prospection, de repérages et d'évaluations des surfaces concernées s'affinant avec le nombre, il s'est constitué peu à peu un corpus de données suffisamment important pour qu'un traitement statistique puisse, en plus des données purement ethnographiques ou techniques, être mis en œuvre. En effet, au total, sur cinq atolls, parmi les plus grands des Tuamotu, qui ont été totalement recensés, ce sont près de 1200 fosses qui ont été décomptées, cartographiées et mesurées dans leurs principales dimensions.

La distribution des valeurs - qu'elles soient arithmétiques, géométriques ou spatiales, pour se limiter aux paramètres les plus élémentaires étant loin d'être homogène, montre que le système aussi bien technique que social qui a régi l'implantation puis l'utilisation des fosses au cours du temps a suivi des variations importantes.

Les informations dites "de tradition orale » recueillies sur place auprès d'anciens témoins oculaires ou d'après des récits de seconde ou troisième « oreille » portent essentiellement sur les procédures purement techniques et agronomiques ainsi que sur des expériences de vies familiales remontant au mieux au début $\mathrm{du} \mathrm{Xx}^{\mathrm{e}}$ siècle. Cet aspect de la vie et des activités culturelles des atolls est apparu comme particulièrement sous-valorisé par la communauté ; ce n'est qu'au prix d'efforts importants et de relances persévérantes que ces informations ont pu être extraites de récits personnels dans lesquels les détails n'étaient jamais spontanément exprimés. C'est ainsi l'un des intérêts que l'étude des atolls des Tuamotu aura fait apparaître, qu'une activité dont la mise en œuvre nécessite de très importants efforts physiques, une communauté d'actions en collaboration, des connaissances empiriques indispensables, une persévérance pour sa mise en œuvre, son entretien et sa régénération, est si peu présente tant dans les récits légendaires ou mythiques que dans les souvenirs et la mémoire collective. En effet, dans l'espace des atolls, la terre et les droits fonciers qui lui sont indissociables y ont pourtant au moins la même importance que dans celui des îles hautes. En outre, la mise en œuvre de techniques particulièrement affinées et adaptées à un environnement exceptionnellement limité nécessitent l'intrication de combinaisons tant techniques que cognitives et sociales, réduites et difficilement contournables.

On a ainsi l'impression que si les droits afférents à la terre ont eu, si ce n'est ont encore, une importance déterminante dans l'organisation sociale et ses divers avatars contemporains, le contenu lui-même attribué aux activités qui lui sont rattachées n'est, quant à lui, absolument pas valorisé. Au contraire des activités liées à la pêche, ou à celles affiliées à la mer et au lagon.

Pourtant, la lecture puis l'observation et les rapides enquêtes que j'ai eu l'occasion d'effectuer aux îles Kiribati et Tuvalu en 1989, après m'être déjà bien avancé dans la reconstitution « archéo-ethnographique» des activités rurales des Tuamotu, ont montré que cela n'était pas le cas dans ces archipels, où les productions vivrières font partie des joutes ou de l'émulation sociale collectives.

\section{L'ethnoarchéologie participante, confrontée au réel}

On a là une différence certainement significative de comportement qui rend compte plutôt d'une sorte de choix d'évolution différent entre l'est et l'ouest du Pacifique. Un des indices est apparu lors de la mise en œuvre du programme d'étude mais aussi de réhabilitation de ces fosses de culture qui a pu être mis en place sur l'atoll de Ana'a, où des circonstances jugées favorables s'y 
manifestaient mieux qu'ailleurs. Là, pendant près de quatre ans, nous avons mis en place et entretenu un activisme qui tentait de mettre en action commune divers aspects qui souvent ne sont pas du ressort de la recherche, stricto sensu. En effet, nous avons utilisé, si ce n'est eu recours à toutes les bonnes volontés locales qui pouvaient, et acceptaient le plus souvent, de se manifester dans le maximum de domaines : les services de l'Agriculture, de l'Éducation, de la Mairie, de la Santé, de certaines confréries religieuses ainsi que des particuliers et de leurs familles étendues. En même temps, les tournages d'un documentaire vidéo bénéficiant de l'expérience de P. Auzépy ont stimulé et même souvent alimenté la réflexion et les questionnements de la communauté, non seulement sur l'avenir de son présent mais aussi sur son passé, dont j'ai rendu compte dans quelques articles ponctuels (Chazine, 1990, 1993, 1997).

Il est de fait que la séparation, voire le vide relationnel qui distancie les classes d'âge s'est trouvé partiellement aboli, car les "vieux", ont eu à cette occasion un droit de parole d'autant plus justifié qu'ils étaient là, porteurs d'un savoir particulier que les jeunes ignoraient totalement; à la différence de ce qu'ils avaient jusqu'à présent rejeté, comme révolu ou obsolète. Or bon nombre de leurs connaissances pouvaient justement, à l'occasion de cette remise en valeur des anciennes techniques horticoles des " ancêtres ", être utilisées quasiment sans restriction. La revitalisation de ces méthodes de productions vivrières, outre qu'elles commençaient à faire effectivement leurs preuves en fournissant des tubercules autochtones stricto sensu, a provoqué également des comportements sociaux et techniques imprévus. L'un d'entre eux en particulier mérite qu'on le précise car il établit une relation ou du moins une analogie comportementale avec les homologues insulaires du Pacifique Ouest.

Le représentant local du Service de l'Économie rurale qu'une amicale complicité a rendu encore plus efficace a du coup lancé l'idée d'organiser un « Concours agricole » qui récompenserait, l'année suivante, les plus belles ou volumineuses productions vivrières de l'atoll. Ce concours eut un très grand succès et révéla une capacité insoupçonnée, voire oubliée, des insulaires à se passionner pour les techniques et les pratiques horticoles possibles sur leur île. L'influence et les réseaux des groupes familiaux qui y participèrent se manifestèrent également en occupant immédiatement un nouveau terrain où les marques de prestige, là comme ailleurs, se devaient d'être activées.
L'analogie avec les systèmes de productivités concurrentiels que les Kiribati entretiennent pour la production et les cérémonies, utilisant leurs gigantesques « babai » (Cyrtosperma chamissonis), s'est ainsi rapidement exprimée et correspondait bien avec celles qui ont été observées par A. Di Piazza (1999). On a ainsi eu, au moins temporairement, l'impression qu'une ancienne tradition horticole, combinant technicité et imbrications sociales, pouvait refaire surface et se trouver immédiatement intégrée dans les structures socioculturelle de la communauté.

\section{Conclusion}

Le fait est que la greffe que nous avons tentée n'a pas eu une durée de vie supérieure aux quelques années qui ont suivi sa mise en place. J'ai présenté ailleurs les raisons ou les oppositions de rationalités tant économiques que structurelles qui en avaient provoqué le déclin, dans ses manifestations alimentaires tout au moins.

$\mathrm{Au}$ moins plusieurs préjugés, qui comme tels ont la vie dure, ont quand même commencé à disparaître, à savoir : rien ne pousse aux Tuamotu, il n'y a pas d'eau potable, les insulaires ne savent/sauraient pas cultiver et les primes au coprah le confirment. Ces contrevérités ne sont actuellement plus colportées, du moins par les services de l'Éducation territoriale, et des formes de revival culturel ont commencé à se manifester aux Tuamotu grâce à des actions conjuguées auxquelles manquaient jusqu'à présent des données ou des informations.

$\mathrm{Du}$ point de vue culturel, il en reste, me semble-t-il, une meilleure mise en confiance des insulaires des Tuamotu qui, au-delà de cette expérience trop ponctuelle, savent maintenant de manière indubitable que s'ils ont été ou sont encore de grands marins et pêcheurs à la fois, ils ont tout autant été d'excellents agriculteurs. Eux dont les ancêtres ont su, en coordonnant leurs efforts et leurs savoir-faire, s'assurer les moyens de leur survie alimentaire, ne peuvent plus non plus en faire fi. Les ressources naturelles apparentes des atolls ne l'annonçaient certainement pas et seule la mise en œuvre de ces capacités, une fois combinées entre elles, le leur ont permis. Une démonstration rare où les méthodes de l'archéologie associées pratiquement à celles de l'ethnoarchéologie ont pu être complétées et même corroborées par des analyses physico-chimiques propres à celles de l'agronomie.

C'est donc aussi parce que j'ai gardé à l'esprit nos discussions, tout en poussant plus loin les apports conceptuels de Jacques Barrau, que j'ai 
persévéré dans cette sorte d'ethno-archéologie participante et que je tiens à lui rendre hommage.

\section{RÉFÉRENCES}

BArrau, J., 1961. Subsistence agriculture in Polynesia and Micronesia, Bernice P. Bishop Museum Bulletin 223, Honolulu, 94 p.

Chazine, J.-M, 1977. Prospections archéologiques à Takapoto, Journal de la Société des Océanistes 56-57, pp. 191-215.

-, 1982. Preliminary report on archaeological research, in S. Hatanaka and N. Shibata (eds), Reao Report, Kanazawa Univ., Japon, pp. 267-348.

_, 1985. Les fosses de culture dans les Tuamotu; travaux et perspectives, Journal de la Société des Océanistes 80, pp. 25-32.

—, 1990. Évolution des établissements humains en milieu insulaire des Tuamotu. Notes et Documents de Sciences Humaines n⿳⺈冂13, Publications de l'OrsTOM, Papeete.

_, 1993. Les atolls des Tuamotu, contraintes et ressources de l'environnement, in C. Beck et F. Delort (eds), Pour une Histoire de l'Environnement, CNRSÉditions, Paris, pp. 259-268.

_, 1997. Des atolls et des jardins, essai d'ethnoarchéologie participante, in S. Tcherkezoff et F. Douaire-Marsaudon (dir.), Le Pacifique aujourd'hui, Éditions du CNRS, pp. 245-260, Paris.
ConTE, É, 1990: Archéologie des Tuamotu ; prospection de dix atolls, CPSH (Centre Polynésien des Sciences Humaines), Papeete.

Di Piazza, A, 1999. Migration d'une plante, migration de ses représentations: le taro des marécages (Cyrtosperma chamissonis) sur Nikunau et Tabuaeran (Kiribati), JATBA, Vol.41, nº1, pp. 93-108.

—, 2001. L'archéologie du voyage; tout un archipel pour territoire, Archeologia 383, pp. 28-37.

EMORy, K.P., 1934. Tuamotuan stone structures, Bernice P. Bishop Museum Bulletin 118, Honolulu.

—, 1975. Material culture in the Tuamotu archipelago, Bernice P. Bishop Museum Bulletin Pacific Anthropological Records, 22, Honolulu.

GARANGER, J. et A. Lavondes, 1966. Recherches archéologiques à Rangiroa, archipel des Tuamotu, Journal de la Société des Océanistes 22, pp. 25-66.

GuILCHER, A., 1967. Les îles Gilbert, comparées aux Tuamotu, Journal de la Société des Océanistes 23, pp. 101-114.

NitTA, Eiji, 1982. Archaeological research, in S. Hatanaka and N. Shibata (eds) Reao Report, Kanazawa Univ., Japon., pp. 348-430.

Otтino, P., 1972. Rangiroa, parenté étendue, résidence et terres dans un atoll polynésien, Éd. Cujas, Paris.

Sinoto, Y., 1976. Preliminary report of archaeological survey on Reao atoll, in S. Hatanaka (ed), A study of the Polynesian migration to Eastern migrations, Kanazawa Univ., pp.102-181.

VAyda, A. P. Ed., 1968. Peoples of the Pacific, Clarendon Univ. Press, New York. 